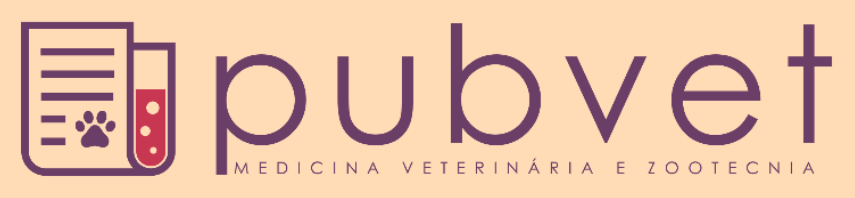

https://doi.org/10.31533/pubvet.v13n2a271.1-7

\title{
Utilização dos restos culturais e resíduos da industrialização de abacaxi na alimentação de ruminantes: Revisão
}

\author{
Kaio Sant'Anna de Paula ${ }^{1 *}$, Oscar Lopes de Faria Júnior ${ }^{20}$ \\ ${ }^{1}$ Acadêmico do instituto Federal Goiano - Campus Ceres. Ceres - Go Brasil \\ ${ }^{2}$ Professor do Instituto Federal Goiano - Campus Ceres. Ceres - GO Brasil. \\ Autor para correspondência, E-mail: kaiodepaula26@hotmail.com
}

\begin{abstract}
Resumo. Do abacaxizeiro é aproveitado apenas $22,5 \%$, correspondente à parte comestível e industrializável, do restante, $4,5 \%$ constituem a casca do fruto e $73 \%$ a parte vegetativa (folhas, caule e coroa), considerados resíduos culturais. Esses resíduos culturais podem ser utilizados na forma de silagem ou feno, proporcionam ganho de peso e ou aumento na produção leiteira, além de minimizar custos e impactos ambientais em regiões produtoras de abacaxi. A industrialização da fruticultura tropical tem propiciado enormes sobras de subprodutos, que in natura ou beneficiados, poderão contribuir com uma parcela expressiva na alimentação de ruminantes. $\mathrm{O}$ uso desses subprodutos é uma alternativa viável e com alto potencial para o uso na alimentação de ruminantes, com ganho de peso diário em ovinos de $0,231 \mathrm{~kg}$ utilizando dieta de $50 \%$ feno de resíduo de abacaxi $+50 \%$ feno de maniçoba, e não alterando o desempenho de bovinos terminados em confinamento com substituição de 20 a $60 \%$ da silagem de milho por silagem de resíduos industriais de abacaxi. Concluindo assim que os resíduos de abacaxi é uma fonte viável de volumoso para ruminantes, porém as pesquisas e as informações da utilização dessas fontes alternativas para alimentação animal ainda são limitadas. O presente estudo tem como objetivo mostrar as formas de utilização dos restos culturais do abacaxi na alimentação de ruminantes
\end{abstract}

Palavras-chave: alimento alternativo, silagem, subprodutos, volumoso

\section{Use of cultural remains and residues of pineapple industrialization in ruminant feed: Review}

\begin{abstract}
Only 22.5\% of the remaining pineapple is harvested, corresponding to the edible and to industry part, the remaining $4.5 \%$ constitute the fruit's bark and $73 \%$ the vegetative part (leaves, stem and crown), considered as cultural waste. These cultural residues can be used in the form of silage or hay, provide weight gain and / or increase in milk production, and minimize environmental costs and impacts in pineapple producing regions. The industrialization of tropical fruit trees has provided enormous leftovers of by-products, which in natura or benefited, may contribute an expressive share in the feeding of ruminants. The use of these by-products is a viable alternative with a high potential for use in ruminant feed, with daily gain in sheep of $0.231 \mathrm{~kg}$ using a $50 \%$ hay diet of pineapple residue $+50 \%$ Manihot pseudoglaziovii hay, and not altering performance of confined finishing cattle with replacement of 20 to $60 \%$ of corn silage by industrial pineapple residue silage. Concluding therefore that pineapple residues are a viable source of bulk for ruminants, however, research and information on the use of these alternative sources for animal feed is still limited. The present study aims to show the ways of using the cultural remains of pineapple in the feeding of ruminants.
\end{abstract}

Key words: bulky, by-products, alternative food, silage 


\title{
Utilización de los residuos agroindustriales de piña en la alimentación de rumiantes: Revisión
}

\begin{abstract}
Resumen. De la piña es aprovechado apenas $22,5 \%$, \%, correspondiente a la parte comestible e industrializable, del restante, $4,5 \%$ constituyen la cascara de la fruta e $73 \%$ la parte vegetativa (hojas, tallo y corona), considerados residuos agroindustriales. Esos residuos pueden ser utilizados en forma de ensilaje o heno, proporcionan una ganancia de peso o un aumento en la producción lechera, además de minimizar costos e impactos ambientales en regiones productoras de piña. La industrialización de la fruticultura tropical ha propiciado enormes sobras de subproductos, que in natura o beneficiados, podrán contribuir con una parte expresiva en la alimentación de rumiantes. El uso de estos subproductos es una alternativa viable y con alto potencial para el uso en la alimentación de rumiantes, con ganancia de peso diario en ovinos de 0,231 kg utilizando dieta de 50\% heno de residuo de piña $+50 \%$ heno de Manihot pseudoglaziovii, y no alterando el rendimiento de bovinos engordados en corral con sustitución de 20 a $60 \%$ del ensilaje de maíz por ensilaje de residuos industriales de piña. Concluyendo que los residuos de piña son una fuente viable de fibroso para rumiantes, pero las investigaciones y la información sobre la utilización de estas fuentes alternativas para la alimentación animal siguen siendo limitadas. El presente estudio tiene como objetivo mostrar las formas de utilización de los residuos de la cosecha de piña en la alimentación de rumiantes.
\end{abstract}

Palabras clave: alimento alternativo, ensilaje, subproductos, voluminoso

\section{Introdução}

A agricultura e a pecuária representam grande parte do PIB nacional. Segundo o Instituto Brasileiro de Geografia e Estatística (IBGE) representaram em torno de 23\% do PIB em 2017. Sendo o Brasil o maior produtor de abacaxi da América Latina ocupando o terceiro lugar na produção mundial. Em busca de melhorar a produção de abacaxi e a qualidade dos frutos, o uso de tecnologias e formas de aproveitamento dos restos culturais tem aumentado cada dia mais, com a finalidade de minimizar os impactos ambientais e ainda prevenir doenças na lavoura.

A industrialização da fruticultura tropical tem propiciado enormes sobras de subprodutos, que in natura ou beneficiados poderiam contribuir com uma parcela expressiva na alimentação de ruminantes (Cunha et al., 2009; Santos et al., 2014). A industrialização do abacaxi possui dois tipos de resíduos, o resíduo da industrialização e o resíduo cultural. Ambos os resíduos podem ser utilizados na alimentação de ruminantes na forma de silagem (Lallo et al., 2003). Os restos produzidos pela cultura do abacaxi podem ainda ser incorporados ao solo para o plantio da próxima cultura (Silva, 2014).

O resíduo da indústria é composto pela prensagem de subprodutos como cascas, talos, coroas e cilindro e o resíduo cultural é composto por folhas, caules e raízes. O resíduo pós-colheita da planta de abacaxi é uma fonte de forragem que possui uso limitado, entretanto, apresenta potencial para o aumento da produção animal nos locais onde são cultivados (Marin et al., 2002). Da planta do abacaxi pode-se obter o farelo, que é palatável e altamente digestível, rico em carboidrato, pobre em proteína e apresenta teor elevado de fibra bruta, destacando-se como fonte de energia para ruminantes (Marin et al., 2002).

Estudos e trabalhos atuais com o aproveitamento dos restos culturais do abacaxi têm mostrado resultados muito promissores com o uso da silagem como forma de armazenagem da forrageira e com bons resultados de seu uso na alimentação de ruminantes (Fagundes \& Fagundes, 2010; Lallo et al., 2003; Prado et al., 2003; Santos et al., 2014). No entanto as informações na literatura ainda são escassas devido ao uso da planta de abacaxi na alimentação animal ser de forma empírica. O presente estudo tem como objetivo mostrar as formas de utilização dos restos culturais do abacaxi na alimentação de ruminantes.

\section{Revisão de literatura}

A intensificação dos sistemas de exploração exige uma maior utilização de insumos alimentícios, não só para cobrir os períodos críticos do ciclo anual de produção de forragens, mas também para conseguir uma melhor expressão do potencial genético dos ruminantes (Fagundes \& Fagundes, 2010). 
Segundo Carvalho et al. (1991), apenas 22,5\% do abacaxizeiro é aproveitado, correspondente à parte comestível e industrializável, do restante, $4,5 \%$ constituem a casca do fruto e $73 \%$ a parte vegetativa (folhas, caule e coroa), considerados resíduos culturais. Após a colheita do fruto, há uma grande quantidade de resto cultural, com potencial para aumentar a produção animal que, muitas vezes, por falta de informações, acaba sendo inutilizado e desperdiçado (Lallo et al., 2003). Os restos culturais de abacaxi poderiam servir como alimento na forma de silagem, inclusive contribuindo para baixar o custo da ração para ruminantes. A silagem de resíduos industriais de abacaxi, por apresentar características nutricionais próximas à da silagem de milho, sua composição em PB seria semelhante à silagem de milho $(6,50$ a $7,05 \%)$ poderia substituí-la como fonte de volumoso para animais em confinamento. Além da qualidade nutricional é um produto de baixo custo por ser considerado um resíduo, diferente de outros frequentemente utilizados, como por exemplo, a silagem de milho, que apresenta altos valores no período de entressafra do milho, sendo que neste período também há queda na produção de forragem. Todavia, esse teor varia em função do material coletado e do período de utilização (Prado et al., 2003).

O alto teor de umidade é um fator limitante para a utilização dos resíduos da agroindústria de frutas, e o custo para desidratar é bastante elevado, sendo o processo de ensilagem uma alternativa viável, porém, devido suas características o processo de ensilagem convencional apresenta perdas significativas (Cunha et al., 2009).

Segundo Marin et al. (2002) além do uso da própria planta verde na alimentação de ruminantes, os restos culturais do abacaxizeiro podem ser usados na forma dessecada (feno de abacaxi), moída (farinha de abacaxi) e em relação à planta, ainda pode-se obter o farelo, que é palatável e altamente digestível, rico em carboidrato, pobre em proteína e por ser muito rico em fibra bruta é útil como fonte de energia para ruminantes. Ferreira et al. (2009) e Alves et al. (2016) evidenciaram que a silagem dos restos culturais do abacaxizeiro mostra-se como boa alternativa forrageira para dietas de ruminantes.

\section{Obtenção e comercialização dos restos culturais de abacaxi para ensilagem}

O uso de silagens de restos culturais é uma alternativa viável ao produtor para a redução dos custos da alimentação assim como uma forma de minimizar a contaminação ambiental com a grande quantidade de resíduos vegetais produzidos (Santos et al., 2010). Como o fruto é a parte comercializável da planta do abacaxi, as folhas, caules e raízes são os restos da planta pós-colheita. A silagem de restos culturais de abacaxi consiste em se fazer a picagem com ensiladeira e a compactação em silos tipo trincheira ou de superfície. Os restos culturais da lavoura poderão ser revendidos ao produtor de leite através de um intermediário que se encarrega do corte, assim como de sua colocação na propriedade rural. Poucos se utilizam da integração agricultura-pecuária, ou seja, poucos agropecuaristas fazem o plantio da lavoura de abacaxi para o aproveitamento dos restos culturais, pois a lavoura ocupa área relativamente grande o que inviabiliza seu plantio para o pequeno produtor de leite (Silva, 2014).

Segundo Silva (2014) como a safra vai de outubro a janeiro e o final do período de espera de crescimento da muda coincide com o final das chuvas, quando o solo permite que a ensiladeira possa entrar na lavoura e proceder a picagem do resto cultural. A fruta pode ser colhida o ano inteiro com ajuda da irrigação, com picos de produção entre outubro e janeiro. Normalmente a comercialização do material picado se dá de março/abril até quase no final da seca; muitos produtores enchem o silo duas vezes, antes do início da seca, programando a abertura para, em média, 30 dias depois e no final da seca, de acordo com a necessidade. Com a integração fruticultura-pecuária, obtêm-se volumosos de alto teor nutritivo (ensilagem das sobras do abacaxi), com redução de custos na dieta do gado. Entretanto, é preciso renovar o cultivo de abacaxi após a primeira colheita.

\section{Silagens na alimentação de ruminantes}

Um dos componentes principais da dieta de ruminantes é o volumoso, de composição química e valores nutricionais variáveis, dependendo de diversos fatores como tipo, espécie e variedade da planta forrageira, tipos de processamento e armazenamento, além de clima, maturidade, entre outros (Silva, 2014).

Conforme Dronawat et al. (1966), em experimento realizado com vacas holandesas, utilizando o farelo de abacaxi presente na mesma quantidade em todas as dietas juntamente com feno de abacaxi. As 
vacas recebendo quantidade limitada de farelo de abacaxi não dispuseram de fonte de volumosos suficiente para a manutenção do alto teor de gordura do leite. A inclusão do feno de abacaxi em adição ao farelo de abacaxi aumentou a gordura do leite de 3,2 para 4,3\%. Segundo Marin et al. (2002), o resíduo verde da planta do abacaxi pós-colheita, fornecida na forma de silagem é comparável ao capim Napier fresco, quanto ao valor alimentar e potencial para a produção de leite, contendo de 65 a $74 \%$ de nutrientes digestíveis totais (NDT).

O milho, o sorgo, o milheto e a cana- de-açúcar são as forrageiras mais utilizadas, sendo o milho a melhor opção pelo seu alto valor nutritivo, maior digestibilidade, rendimento de massa verde e boa aceitação pelos animais. Sua utilização se dá pelo fato de sua composição atender os requisitos para a produção de uma boa silagem como teor de matéria seca entre $30 \%$ a $35 \%$, mínimo de $3 \%$ de carboidratos solúveis na matéria original, baixo poder tampão e por proporcionar uma boa fermentação microbiana (Moraes et al., 2013).

Tabela 1. Valores médios de fibra em detergente neutro (\%FDN) e fibra em detergente ácido (\%FDA)

\begin{tabular}{lccc}
\hline Nutrientes & Silagem de restos culturais do abacaxizeiro & Capim Napier & Silagem de milho \\
\hline FDN, $\%$ & 59,82 & 72,27 & 62,6 \\
FDA, $\%$ & 25,87 & 41,89 & 31,9 \\
\hline
\end{tabular}

Fonte: Possenti et al. (2005) Cunha et al. (2009).

Apesar da grande utilização do milho, é uma silagem que apresenta alto custo de produção em períodos de escassez, a obtenção de um alimento alternativo para os animais se torna de grande importância para o sistema de criação intensivo, diminuindo os custos da alimentação.

A utilização de subprodutos da agroindústria na alimentação de ruminantes é uma alternativa viável desde que se observe a viabilidade econômica de sua utilização, a disponibilidade ao longo do ano e o seu valor nutricional. O resíduo industrial precisa ser descartado sem prejuízo ao meio ambiente; passando por tratamentos adequados até estarem enquadrados nos padrões estabelecidos pela legislação ambiental (Silva, 2014).

A silagem de resíduos industriais de abacaxi pode ser considerada um alimento volumoso de considerável teor proteico, sua composição em proteína bruta seria similar à silagem de milho $(6,50 \mathrm{a}$ $7,05 \%$ ). Porém, esse teor varia em função do material coletado e do período de utilização (Fagundes \& Fagundes, 2010; Prado et al., 2003; Santos et al., 2014).

Carvalho et al. (1991) observaram o efeito da época de colheita da planta de abacaxizeiro na composição bromatológica, realizando plantios em duas épocas (abril e outubro). Os autores concluem que as folhas devem ser utilizadas logo após a colheita dos frutos, para ter simultaneamente alta massa foliar com teores proteicos elevados e de carboidratos insolúveis (celulose + hemicelulose + lignina) baixos (Tabela 2).

Tabela 2. Valores médios de celulose, hemicelulose e lignina em diferentes épocas de plantio

\begin{tabular}{|c|c|c|c|}
\hline Dias após a colheita & Celulose \% & Hemicelulose \% & Lignina $\%$ \\
\hline \multicolumn{4}{|c|}{ Abril } \\
\hline 0 & 18,81 & 14,02 & 5,60 \\
\hline 60 & 18,51 & 15,26 & 5,75 \\
\hline 120 & 21,18 & 17,13 & 7,27 \\
\hline \multicolumn{4}{|c|}{ Outubro } \\
\hline 0 & 14,16 & 14,40 & 2,87 \\
\hline 60 & 19,32 & 17,02 & 5,59 \\
\hline 120 & 18,33 & 19,95 & 7,63 \\
\hline
\end{tabular}

Fonte: Carvalho et al. (1991).

Já para o restolho abacaxizeiro triturado em maquina forrageira após 3 dias de sol, Pinto et al. (2005) encontraram $84,12 \%$ de matéria seca, $5,95 \%$ de proteína bruta, $2,54 \%$ de extrato etéreo, $61,06 \%$ fibra 
em detergente neutro, 30,15\% de fibra em detergente ácido, 5,05\% de matéria mineral, 25,24\% de celulose e 2,10\% de lignina, além de encontrar uma energia bruta de $4.193 \mathrm{kcal} / \mathrm{kg}$ de matéria seca.

Silva (2014) obteve valores bem abaixo de proteína bruta (Tabela 3), dos valores encontrados por Prado et al. (2003) em silagem de restos culturais de abacaxi pérola independente dos dias de fermentação e tamanho das partículas, confirmando que esses valores dependem do material coletado e do período de utilização, atingindo o máximo de 5,43\% de PB (proteína bruta) aos 60 dias de fermentação.

Tabela 3. Valores médios em porcentagem, para matéria seca (MS), proteína bruta (PB) e nutrientes digestíveis totais (NDT), em silagem de restos culturais de abacaxi perola.

\begin{tabular}{lccc}
\hline Tratamentos (dias) & MS & PB & NDT \\
\hline 30 & 18,91 & 5,27 & 67,53 \\
60 & 18,16 & 5,43 & 65,80 \\
\hline
\end{tabular}

Fonte: Silva (2014).

Cunha et al. (2009) em estudo com carneiros alimentados com resíduos de abacaxi em forma de silagem (resíduo) e feno utilizando os seguintes tratamentos T1 = Resíduo 60\% + feno de maniçoba 40\%; T2 = Resíduo 50\% + feno de capim 30\% + feno de maniçoba 20\%; T3 = Resíduo 40\% + feno de capim $40 \%$ + feno maniçoba $20 \%$; T4 = Resíduo 35\% + feno capim $45 \%$ + feno maniçoba $20 \%$ e T5 = $50 \%$ feno de resíduo de abacaxi $+50 \%$ feno de maniçoba, o consumo de matéria seca variou de 3,6 a 4,3\% PV e de 80,5 a 95,83 g/UTM (unidade de tamanho metabólico), respectivamente, entre as silagens, enquanto para o feno os valores foram de 4,6\% PV e 102,9 g/UTM, respectivamente. Estes resultados foram superiores aos obtidos por Reis et al. (2000) que trabalhando com silagens mistas $(75 ; 50$ e $25 \%)$ de maracujá na alimentação de ovinos encontraram consumos de 53,85; 60,46 e 52,72 g/UTM, respectivamente, entre as silagens. $O$ peso final não foi influenciado pelas dietas experimentais fornecidas aos animais, sem alterar o desempenho dos animais, indicando que o resíduo usado nas diferentes formas apresentou boa aceitação pelos animais não limitando o consumo. A conversão alimentar da MS não sofreu efeito da dieta, entretanto, T5 mostrou tendência de maior eficiência.

Carvalho et al. (2004) utilizando farelo de cacau em substituição parcial do milho o do farelo de soja $(7 \%, 14 \%$ e $21 \%)$ na dieta obtiveram resultados satisfatórios para ganho de peso diário de 137,86 g, $140,71 \mathrm{~g}$ e 101,53 g, respectivamente, mas sendo inferior aos resultados obtidos por Cunha et al. (2009) utilizando silagem de restos culturais de abacaxi. Suksathit et al. (2011) avaliando efeito dos níveis de resíduos de abacaxi (silagem) e feno de Pangola, $100 \%$ resíduo de abacaxi; $65 \%$ resíduo de abacaxi e $35 \%$ feno de Pangola; $35 \%$ resíduo de abacaxi e $65 \%$ feno de Pangola, como fontes de volumoso para bovinos nativos da Tailândia Meridional, com relação volumoso x concentrado de 65:35. Observou que aumentando as proporções de silagem de resíduos de abacaxi nas dietas, ocorreu uma melhora da digestibilidade dos nutrientes, sem exercer efeito prejudicial à microbiota ruminal, concluindo que a silagem de resíduo de abacaxi se mostra viável como fonte alternativa de volumoso para o sul da Tailândia para o gado nativo comparado ao feno de Pangola. Santos et al. (2014) trabalhando com a substituição da silagem da cana-de-açúcar com os seguintes valores, 25,0\% de MS, 65,0\% de FDN, 4,0 $\%$ de PB, 7,0\% de MM e 8,0\% de lignina e 55,3\% de NDT, pela silagem dos restos culturais de abacaxi com os seguintes valores, $23,60 \%$ de MS; 73,1\% de FDN; 6,3\% de PB; 7,0\% de lignina e 58,0\% de NDT na alimentação de ovinos com cinco tratamentos, $100 \%$ silagem de abacaxi; $75 \%$ silagem de abacaxi e $25 \%$ silagem de cana-de-açúcar; $50 \%$ silagem de abacaxi e 50\% silagem de cana-de-açúcar; $25 \%$ silagem de abacaxi e $75 \%$ silagem de cana-de-açúcar e $100 \%$ silagem de cana-de-açúcar, com razão volumoso e concentrado de 60 e 40 . Concluiu que em função da taxa de desaparecimento de MS do rúmen, digestibilidade e consumo de MS pode-se utilizar a silagem de restos culturais do abacaxizeiro em substituição à silagem de cana-de-açúcar $+0,5 \%$ ureia na alimentação de ovinos.

Rodas et al. (1991) trabalhando com silagem de abacaxi na alimentação de bovinos em terminação afirmaram que não houve diferença para consumo de matéria seca; no entanto, o consumo de energia metabolizável, ganho de peso diário e conversão alimentar foi melhor para os animais expostos ao alto nível de proteína, das dietas suplementadas com pasta de girassol, melaço e ureia para aumentar o nível proteico da silagem de abacaxi. Mostrando assim que a silagem pode ser complementada com adição 
de outras fontes de proteína para melhorar o desempenho dos animais alimentados com silagem de abacaxi. Todavia, segundo Rodríguez-Chacón et al. (2014) deve-se avaliar a adição dessa suplementação com aditivos, como a ureia e o melaço a nível ruminal para se otimizar o uso de cada recurso afim de se identificar a forma mais eficiente dos aditivos.

Por apresentar composição química e características fermentativas favoráveis o uso da silagem de resíduos industriais de abacaxi como alimento alternativo na terminação de bovinos de corte com níveis de substituição de 20 a $60 \%$ da silagem de milho se mostra favorável não alterando o desempenho animal, a conversão alimentar e o rendimento de carcaça (Lallo et al., 2003; Prado et al., 2003).

\section{Considerações finais}

O uso da silagem e feno de restos culturais e resíduos industriais de abacaxi na alimentação de ruminantes possuem resultados satisfatórios para ganho de peso e conversão alimentar. Sendo uma fonte alternativa de volumoso, diminuindo gastos e sendo uma fonte de fibra nos períodos de estiagem. Podendo ser utilizado tanto para animais de corte como leiteiros. Além de reduzir impactos ambientais e contaminação da próxima lavoura de abacaxi. No entanto, novas pesquisas e trabalhos com o uso desse material e de suma importância para saber qual a melhor forma de armazenamento e forma de oferta aos animais assim como suas características nutricionais e o custo benefício do uso dessa dieta para pequenos e grandes ruminantes.

\section{Referências bibliográficas}

Alves, G. R., Fontes, C. A. A., Fernandes, A. M., Processi, E. F., Oliveira, T. S., Glória, L. S. \& Gomes, R. S. (2016). Intake and digestibility of silages containing pineapple pulp and coast-cross hay with or without urea and markers recovery in sheep. Revista Brasileira de Zootecnia, 45(4):151-157.

Carvalho, G. G. P., Pires, A. J. V., Silva, F. F., Veloso, C. M., Silva, R. R., Silva, H. G. O., . . Souza, S. M. (2004). Comportamento ingestivo de cabras leiteiras alimentadas com farelo de cacau ou torta de dendê. Pesquisa Agropecuária Brasileira, 39(9):919-925.

Carvalho, V. D., Paula, M. B., Abreu, C. M. P. \& Chagas, S. J. R. (1991). Efeito da época de colheita da planta na composição química das folhas do abacaxizeiro. Pesquisa Agropecuária Brasileira, 26(10):1655-1661.

Cunha, M. G. G., Oliveira, E. R., Ramos, J. L. F. \& Alcântara, M. D. B. (2009). Conservação e utilização do resíduo de abacaxi na alimentação de ovinos no Curimataú Ocidental da Paraíba. Tecnologia \& Ciência Agropecuária, 3(3):55-62.

Dronawat, N. S., Stanley, R. W., Cobb, E. \& Morita, K. (1966). Effect of feeding limited roughage and a comparison between loose and pelleted pineapple hay on milk production, milk constituents, and fatty acid composition of milk fat. Journal of Dairy Science, 49(1):28-31.

Fagundes, N. S. \& Fagundes, N. S. (2010). Restos culturais do abacaxizeiro na alimentação de ruminantes. Revista Eletrônica Nutritime, 71243 - 1247.

Ferreira, A. C. H., Neiva, J. N. M., Rodriguez, N. M., Campos, W. E. \& Borges, I. (2009). Avaliação nutricional do subproduto da agroindústria de abacaxi como aditivo de silagem de capim-elefante. Revista Brasileira de Zootecnia, 38(2):223-229.

Lallo, F. H., Prado, I. N., Nascimento, W. G., Zeoula, L. M., Moreira, F. B. \& Wada, F. Y. (2003). Substitution levels of corn silage by pineapple by-products on ruminal degradability in beef cattle. Revista Brasileira de Zootecnia, 32(3):719-726.

Marin, C. M., Suttini, P. A., Sanches, J. P. F. \& Bergamaschine, A. F. (2002). Potencial produtivo e econômico da cultura do abacaxi e o aproveitamento de seus subprodutos na alimentação animal. Ciências Agrarias e da Saúde, 2(1):79-82.

Moraes, S. D., Jobim, C. C., Silva, M. S. \& Marquardt, F. I. (2013). Produção e composição química de híbridos de sorgo e de milho para silagem. Revista Brasileira de Saúde e Produção Animal, 14(4):624-634.

Pinto C. W. C., Sousa, W. H., Pimenta Filho, E. C., Cunha, M. das G. G. \& Gonzaga Neto, S. (2005) Desempenho de cordeiros Santa Inês terminados com diferentes fontes de volumosos em confinamento. Agropecuária Técnica, Areia, 26(2), p. 123-128. 
Possenti, R. A., Ferrari Júnior, E., Bueno, M. S., Bianchini, D., Leinz, F. F. \& Rodrigues, C. F. (2005). Bromatological and fermentative parameters of corn and sunflower silages. Ciência Rural, 35(5):1185-1189.

Prado, I. N., Lallo, F. H., Zeoula, L. M., Caldas Neto, S. F., Nascimento, W. G. \& Marques, J. A. (2003). Bulls performance in feedlot with levels of substituting corn silage by pineapple by-products silage. Revista Brasileira de Zootecnia, 32(3):737-744.

Reis, J., Paiva, P. C. A., Tiesehausen, I. M. E. V. V. \& Resende, G. A. P. (2000). Composição química, consumo voluntário e digestibilidade de silagens de resíduos do fruto de maracujá (Passiflora edulis Sims f. flavicarpa) e de capim-elefante (Pennisetum purpureum Schum) cv. Cameroon e suas combinações. Ciência e Agrotecnologia, 24(1):213-224.

Rodas, E. V., Ortíz, G. A. O., Vadillo, J. C. V. \& Lagunes, H. V. B. (1991). Utilización del ensilaje de planta de piña (ananas comosus) en la aumentación de bovinos en confinamiento. Revista Mexicana de Ciencias Pecuarias, 29(3):111-116.

Rodríguez-Chacón, S., López-Herrera, M., WingChing-Jones, R. \& Rojas-Bourillón, A. (2014). Adición de melaza deshidratada y urea en ensilados de rastrojos de piña. Agronomía Mesoamericana, 25(2):313-321.

Santos, M., Gómez, A., Perea, J., García, A., Guim, A. \& Pérez, M. (2010). Fatores que afetam o valor nutritivo da silagens de forrageiras tropicais. Archivos de Zootecnia, 5925-43.

Santos, S. C., Fernandes, J. J. R., Carvalho, E. R., Gouvea, V. N., Lima, M. M. \& Dias, M. J. (2014). Utilização da silagem de restos culturais do abacaxizeiro em substituição à silagem de cana-de-açúcar na alimentação de ovinos. Ciência Animal Brasileira, 15(4):400-408.

Silva, M. L. S. (2014). Avaliação nutricional de silagem de restos culturais de abacaxi pérola. Universidade estadual paulista - unesp. Jaboticabal, p. 50. . Jaboticabal, São Paulo, Brasil: Universidade Estadual Paulista.

Suksathit, S., Wachirapakorn, C. \& Opatpatanakit, Y. (2011). Effects of levels of ensiled pineapple waste and pangola hay fed as roughage sources on feed intake, nutrient digestibility and ruminal fermentation of Southern Thai native cattle. Songklanakarin Journal of Science \& Technology, 33(3):281-289.

Recebido: 5 de dezembro, 2019.

Aprovado: 17 de janeiro, 2019

Publicado: 26 fevereiro, 2019.

Licenciamento: Este artigo é publicado na modalidade Acesso Aberto sob a licença Creative Commons Atribuição 4.0 (CC-BY 4.0), a qual permite uso irrestrito, distribuição, reprodução em qualquer meio, desde que o autor e a fonte sejam devidamente creditados. 